\title{
INTEGRAL EQUATIONS FOR SPECIAL FUNCTIONS OF HEUN CLASS
}

\author{
A. Ya. Kazakov and S. Yu. Slavyanov
}

\begin{abstract}
Integral equations equivalent to boundary problems for the differential equations of Heun class are studied. A new method of their derivation is proposed which is not as general as the known ones but is efficient in creating integral equations of a certain type. A number of new integral equations are obtained, some related to the double-confluent Heun equation. Formal calculations at the first stage are followed by rigorous formulations.
\end{abstract}

\section{Introduction}

The well-known special functions of mathematical physics, Bessel functions, hypergeometric functions, etc., are defined as solutions of linear ordinary second-order differential equations with polynomial coefficients. Below we call any solution of such equations a special function. The special functions can be expressed by means of integral representations in terms of elementary functions. These representations are of particular importance for further study of additional characteristics of these functions: recurrence relations, asymptotic expansions, etc.

There is no hope of finding such representations for all special functions in our more general sense; however, there exist more sophisticated integral relations and integral equations which do succeed. Our main goal in this article is to deduce several simple integral equations for some special functions, namely of Heun type and the corresponding confluent cases $[4,10]$. These functions usually arise as eigenfunctions of an eigenvalue problem associated with the initial differential equation.

Several of our results are known. The main tools used previously for their derivation are either utilization of some integral transforms [6] or separation of variables in an auxiliary partial differential equation (pde) [1,2, 5, 7-9]. Our approach is based on the polynomial structure of the original equation, and it permits us to obtain new relations as well as to give a new derivation of the old ones in a unique manner. On the other hand, it does not give the large variety of relations depending on an additional parameter obtained by separation of variables of the mentioned pde. In fact these two methods are complementary to each other.

\section{Major definitions}

By a Heun equation we mean a second-order linear homogeneous differential equation with four regular singular points. With the help of a linear transformation of the independent variable these points can be located at $z_{1}=0, z_{2}=1, z_{3}=a, z_{4}=\infty$ with the arbitrary parameter $a$. A simple transformation of the dependent variable may further convert the given equation to the so-called canonical form [4]

$$
L_{z}^{(0)}(a, \alpha, \beta, \gamma, \delta, q) y(z)=0
$$

Received October 29, 1993, revised March 15, 1996.

1991 Mathematics Subject Classification: 44A10, 44A15, 44A20.

Key words and phrases: Heun equation, confluent cases, integral equations. 


$$
\begin{gathered}
L_{z}^{(0)}=z(z-1)(z-a) D^{2}+(\gamma(z-1)(z-a)+\delta z(z-a) \\
+(\alpha+\beta+1-\gamma-\delta) z(z-1)) D+(\alpha \beta z+q),
\end{gathered}
$$

where $D=\frac{d}{d z}$. Here $a, \alpha, \beta, \gamma, \delta, q$ may be arbitrary (generally complex) parameters. However, we shall pay major attention to the case when they are real and, moreover, satisfy additional conditions.

In the vicinity of each regular singularity $z_{j}$ of equation (2), there exist two solutions $y_{m}\left(z_{j}, z\right), m=1,2$ determined by the behaviour at the singularity:

$$
\begin{aligned}
& y_{1}\left(z_{j}, z\right)=\left(z-z_{j}\right)^{\rho_{1}\left(z_{j}\right)}\left(1+O\left(z-z_{j}\right)\right), \\
& y_{2}\left(z_{j}, z\right)=\left(z-z_{j}\right)^{\rho_{2}\left(z_{j}\right)}\left(1+O\left(z-z_{j}\right)\right), \quad j=1,2,3 \\
& y_{1}(\infty, z)=z^{-\rho_{1}(\infty)}\left(1+O\left(z^{-1}\right)\right) \\
& y_{2}(\infty, z)=z^{-\rho_{2}(\infty)}\left(1+O\left(z^{-1}\right)\right)
\end{aligned}
$$

They are called Frobenius solutions. The numbers $\rho_{m}\left(z_{j}\right)(m=1,2 ; j=1,2,3)$ are called characteristic exponents at the singularity $z_{j}$. In case of the canonical form of the Heun equation, $\rho_{1}\left(z_{j}\right)=0$. The numbers $\rho_{1}(\infty), \rho_{2}(\infty)$ are called characteristic exponents at infinity. Formulas (3)-(4) must be modified in the case when the difference $\rho_{1}\left(z_{j}\right)-\rho_{2}\left(z_{j}\right)$ is an integer. The study presented below also needs special care in this case; this is not always stressed. Characteristic exponents $\rho_{2}\left(z_{j}\right), j=1$, $2,3, \rho_{1}(\infty), \rho_{2}(\infty)$ are easily expressed explicitly in terms of parameters $\alpha, \beta, \gamma, \delta$, and do not depend upon the parameter $q$, which is called the accessory parameter of equations (1)-(2):

$$
\begin{aligned}
& \rho_{2}(0)=1-\gamma, \quad \rho_{2}(1)=1-\delta, \quad \rho_{2}(a)=\gamma+\delta-\alpha-\beta \\
& \rho_{1}(\infty)=\alpha, \quad \rho_{2}(\infty)=\beta
\end{aligned}
$$

We suppose that $\rho_{1}\left(z_{j}\right), \rho_{2}\left(z_{j}\right)$ are real and

$$
\rho_{2}\left(z_{j}\right)<0, \quad j=1,2,3,
$$

which implies certain restrictions on $\alpha, \beta, \gamma, \delta$. In addition to the operator $L_{z}^{(0)}$, the formally adjoint operator $L_{z}^{(0) *}$ can be defined by

$$
\begin{aligned}
L_{z}^{(0) *}= & D^{2} z(z-1)(z-a)-D(\gamma(z-1)(z-a)+\delta z(z-a) \\
& \quad+(\alpha+\beta+1-\gamma-\delta) z(z-1))+\alpha \beta z+q \\
= & L_{z}^{(0)}(a, 2-\alpha, 2-\beta, 2-\gamma, 2-\delta, \hat{q}), \\
\hat{q}= & q-\gamma a-\delta(a-1)-\alpha-\beta+2 a+1 .
\end{aligned}
$$

Solutions corresponding to the equation

$$
\left(L_{z}^{(0)}\right)^{*} v(z)=0
$$

are characterized by characteristic exponents $\rho_{m}^{*}\left(z_{j}\right)$, which satisfy

$$
\rho_{1}^{*}\left(z_{j}\right)=0, \quad \rho_{2}^{*}\left(z_{j}\right)=-\rho_{2}\left(z_{j}\right), \quad j=1,2,3 .
$$

Confluent cases of the Heun equation arise when regular singular points coalesce so as to form irregular singular points [4]. The following equations can be distinguished by general values of the parameters.

Firstly, the confluent Heun equation

$$
\begin{aligned}
& L_{z}^{(1)} y=0 \\
& L_{z}^{(1)}=z(z-1) D^{2}+(-p z(z-1)+\gamma(z-1)+\delta z) D+(-p \alpha z+q),
\end{aligned}
$$


is characterized by two regular singularities at $z_{1}=0, z_{2}=1$ and one irregular singularity at infinity. Once more Frobenius solutions can be defined in the vicinities of regular singularities characterized by characteristic exponents $\rho_{1}(0)=0, \rho_{2}(0)=1-\gamma$, $\rho_{1}(1)=0, \rho_{2}(1)=1-\delta$. We suppose that

$$
\rho_{2}\left(z_{i}\right)<0, \quad j=1,2 .
$$

At infinity, other types of solutions, often called Thome solutions, can be defined. They are characterized by asymptotic behavior of the form

$$
y_{m}(z) \sim z^{\alpha_{m}(\infty)} \exp \left(\beta_{m}(\infty) z\right), \quad m=1,2,
$$

in appropriate sectors where the $\beta_{m}(\infty)\left(\beta_{1}(\infty)=0, \beta_{2}(\infty)=p\right)$ and $\alpha_{m}(\infty)$ $\left(\alpha_{1}(\infty)=\alpha, \alpha_{2}(\infty)=\gamma+\delta-\alpha\right)$ are called characteristic exponents of the second kind. We suppose that

$$
\beta_{2}(\infty)<0
$$

Secondly, we obtain the biconfluent Heun equation

$$
L_{z}^{(2)} y=0, \quad L_{z}^{(2)}=z D^{2}+\left(-z^{2}-\mu z+\gamma\right) D+(-\alpha z+q),
$$

with one regular singularity at zero and one irregular singularity at infinity. Thome solutions at infinity are characterized by the asymptotics:

$$
y_{m}(z) \sim z^{-\alpha_{m}(\infty)} \exp \left(\frac{\gamma_{m}(\infty) z^{2}}{2}+\beta_{m}(\infty) z\right), \quad m=1,2
$$

with characteristic exponents of the second kind $\alpha_{m}(\infty)\left(\alpha_{1}(\infty)=\alpha, \alpha_{2}(\infty)=1+\right.$ $\gamma-\alpha), \beta_{m}(\infty)\left(\beta_{1}(\infty)=0, \beta_{2}(\infty)=\mu\right)$, and $\gamma_{m}(\infty)\left(\gamma_{1}(\infty)=0, \gamma_{2}(\infty)=1\right)$.

The so-called double-confluent Heun equation can be presented in the form

$$
L_{z}^{(3)} y=0, \quad L_{3}^{(3)}=z^{2} D^{2}+\left(-z^{2}+\tau z+\nu\right) D-\alpha z+q
$$

with two irregular singularities: one at zero and the other at infinity. At infinity, Thome solutions resemble (12), and near zero we have

$$
y_{m}(z) \sim z^{\alpha_{m}(0)} \exp \left(\frac{\beta_{m}(0)}{z}\right), \quad m=1,2,
$$

with $\beta_{1}(0)=0, \beta_{2}(0)=\nu, \alpha_{1}(0)=0$, and $\alpha_{2}(0)=2-\tau$.

Finally, the triconfluent Heun equation is

$$
L_{z}^{(4)} y=0, \quad L_{z}^{(4)}=D^{2}+\left(-z^{2}-\sigma\right) D-\alpha z+q .
$$

Equation (18) is characterized by only one irregular singularity located at infinity, which appears as a result of confluence of three regular singularities. Thome solutions at infinity are characterized by the asymptotic behavior:

$$
y_{m}(z) \sim z^{\alpha_{m}(\infty)} \exp \left(\frac{\delta_{m}(\infty) z^{3}}{3}+\frac{\gamma_{m}(\infty) z^{2}}{2}+\beta_{m}(\infty) z\right), \quad m=1,2,
$$

with characteristic exponents of the second kind $\alpha_{m}(\infty)\left(\alpha_{1}(\infty)=\alpha, \alpha_{2}(\infty)=2-\alpha\right)$ $\beta_{m}(\infty)\left(\beta_{1}(\infty)=0, \beta_{2}(\infty)=\sigma,\right), \gamma_{m}(\infty)\left(\gamma_{1}(\infty)=\gamma_{2}(\infty)=0,\right)$, and $\delta_{m}(\infty)$ $\left(\delta_{1}(\infty)=0, \delta_{2}(\infty)=1\right)$. We discuss this equation only for completeness since there is no integral equation of Fredholm type related to this equation.

Besides the confluent equations listed above, several additional equations can be studied. They arise by means of a slightly modified confluent process. At first, one of the regular singularities $z_{j}$ by specialization of parameters is reduced to an elementary singularity for which $\rho_{2}\left(z_{j}\right)=1 / 2$. Further irregular singularities appear as a result of the confluence process with participation of this elementary point. With the help of 
such procedures, we get four more equations of Heun type which, as far as we know, have no conventional names:

$$
\begin{array}{ll}
L_{z}^{(5)} y(z)=0, & L_{z}^{(5)}=z(z-1) D^{2}+(\gamma(z-1)+\delta z) D-k^{2} z+q, \\
L_{z}^{(6)} y(z)=0, & L_{z}^{(6)}=z D^{2}+\gamma D+\left(-z^{2}+f z+q\right), \\
L_{z}^{(7)} y(z)=0, & L_{z}^{(7)}=z^{2} D^{2}+(\rho z-\omega) D-z+q, \\
L_{z}^{(8)} y(z)=0, & L_{z}^{(8)}=D^{2}+\left(-z^{3}+f z+q\right) .
\end{array}
$$

Equations (1), (10), (14), (16), (18), (20)-(23) constitute an essential Heun class of equations in the sense that their derivation is based not on simple substitution but on a certain limiting process. All of these equations may be presented in general as follows:

$$
L y=0, \quad L=R(z) D^{2}+P(z) D+Q(z)
$$

with different polynomials $R(z), P(z)$, and $Q(z)$, all of them being not higher than of the third degree. Among the coefficients of these polynomials, an exceptional role is played by the accessory parameter $q$, which does not influence the characteristic exponents at the singularities. Taking this parameter as a spectral one, we can turn equation (24) by the transformation

$$
y(z)=R^{1 / 2} \exp \left(-\int \frac{P}{2 R} d z\right), \quad w(z)=G(z) w(z),
$$

into the self-adjoint form

$$
D R(z) D w(z)+(\tilde{Q}(z)+q) w(z)=0
$$

where

$$
\tilde{Q}(z)=\frac{R^{\prime \prime}-P^{\prime}}{2}-\frac{\left(P-R^{\prime}\right)^{2}}{4 R}+Q-q .
$$

Taking the enumerated operators $L_{z}^{(j)}, j=0, \ldots, 8$, we transform them by means of (24) to self-adjoint operators $M_{z}^{(j)}$,

$$
M_{z}^{(j)}=\left[G^{(j)}(z)\right]^{-1} L_{z}^{(j)} G_{z}^{(j)}-q=M_{z}^{(j) *}
$$

corresponding to the equation

$$
\left(M_{z}^{(j)}+q\right) w(z)=0, \quad j=0, \ldots, 8 .
$$

The functions $G^{(j)}(z)$ can be expressed either in terms of the parameters of the original equation or in terms of characteristic exponents. The second possibility looks more representative. For instance, we have

$$
\begin{aligned}
& G^{(0)}(z)=z^{\rho_{2}(0) / 2}(1-z)^{\rho_{2}(1) / 2}(z-a)^{\rho_{2}(a) / 2} \\
& G^{(1)}(z)=z^{\rho_{2}(0) / 2}(1-z)^{\rho_{2}(1) / 2} \exp \left(\frac{\beta_{2}(\infty) z}{2}\right), \\
& G^{(2)}(z)=z^{\rho_{2}(0) / 2} \exp \left(\frac{\gamma_{2}(\infty) z^{2}}{4}+\frac{\beta_{2}(\infty) z}{2}\right), \\
& G^{(3)}(z)=z^{\alpha_{2}(0) / 2} \exp \left(\frac{\beta_{2}(\infty) z}{2}+\frac{\beta_{2}(0)}{2 z}\right) .
\end{aligned}
$$

Functions $G^{(j)}(z)$ relating to operators (20)-(23) are discussed below. 


\section{Formal computations}

In this section, we shall obtain the major results of the article on a formal level. We seek an integral relation of the form

$$
y(z)=\int_{C} A(z, t) v(t) d t=A v
$$

where $y(z)$ is a solution of equation $(24)$ and $v(t)$ is a solution of the adjoint equations and we check that some formal commutation rule holds. To be rigorous, we have to check in addition that

(i) non-integral terms arising from integration by parts vanish,

(ii) the functions $y(z), v(z)$ and the kernels belong to the proper spaces of functions,

(iii) the required behavior at singularities is reproduced by (34), and

(iv) all integrals converge.

These additional requirements imply certain conditions for the parameters of the initial equation which for simplicity have been partly formulated above. The contour of integration is supposed to be an interval $\left[T_{1}, T_{2}\right]$ on the real axis.

Lemma 1. Suppose that (34) holds and that the function $A(z, t)$ is a solution of the pde

$$
\left(L_{z}-L_{t}\right) A(z, t)=0 .
$$

Let $v(t)$ be a proper solution of $L_{t}^{*} v=0$. Then $y(z)$ is a proper solution of (24).

The proof of this lemma, which is well-known, follows from formal computations which neglect the terms arising from integration by parts.

$$
L_{z} y(z)=\int_{C} v(t) L_{z} A(z, t) d t=\int_{C} v(t)\left(L_{z}-L_{t}\right) A(z, t) d t+\int_{C} A(z, t) L_{t}^{*} v(t) d t
$$

One also can find solutions of (35) by the method of separation of variables. In this article, we present the simplest solutions of (35).

Lemma 2. Suppose that

$$
A(z, t)=A(\xi), \quad \xi=z+t .
$$

Then the operators $L_{z}^{(i)}-L_{t}^{(i)}, i=1, \ldots, 7$, may be represented as

$$
\begin{aligned}
& L_{z}^{(1)}-L_{t}^{(1)}=(z-t)\left((\xi-1) D_{\xi}^{2}+(-p(\xi-1)+\gamma+\delta) D_{\xi}-p \alpha\right), \\
& L_{z}^{(2)}-L_{t}^{(2)}=(z-t)\left(D_{\xi}^{2}-(\mu+\xi) D_{\xi}-\alpha\right), \\
& L_{z}^{(3)}-L_{t}^{(3)}=(z-t)\left(\xi D_{\xi}^{2}+(\tau-\xi) D_{\xi}-\alpha\right), \\
& L_{z}^{(4)}-L_{t}^{(4)}=-(z-t)\left(\xi D_{\xi}+\alpha\right) \\
& L_{z}^{(5)}-L_{t}^{(5)}=(z-t)\left((\xi-1) D_{\xi}^{2}+(\gamma+\delta) D_{\xi}-k^{2}\right), \\
& L_{z}^{(6)}-L_{t}^{(6)}=(z-t)\left(D_{\xi}^{2}-\xi+f\right) \\
& L_{z}^{(7)}-L_{t}^{(7)}=(z-t)\left(\xi D_{\xi}^{2}+\rho D_{\xi}-1\right) .
\end{aligned}
$$

All these equations are proved by direct substitution.

Lemma 3. Suppose that the kernel $A(z, t)$ is of the form

$$
A(z, t)=A(\eta), \quad \eta=z t .
$$


Then the operators $L_{z}^{(i)}-L_{t}^{(i)}, i=0, \ldots, 3,5,7$, may be represented as

$$
\begin{aligned}
& L_{z}^{(0)}-L_{t}^{(0)}=(z-t)\left(\eta(\eta-a) D_{\eta}^{2}+((\alpha+\beta+1) \eta-a \gamma) D_{\eta}+\alpha \beta\right), \\
& L_{z}^{(1)}-L_{t}^{(1)}=(z-t)\left(\eta D_{\eta}^{2}+(-p \eta+\gamma) D_{\eta}-p \alpha\right), \\
& L_{z}^{(2)}-L_{t}^{(2)}=-(z-t)\left(\eta D_{\eta}^{2}+(\eta+\gamma) D_{\eta}+\alpha\right), \\
& L_{z}^{(3)}-L_{t}^{(3)}=-(z-t)\left((\eta+\nu) D_{\eta}+\alpha\right), \\
& L_{z}^{(5)}-L_{t}^{(5)}=(z-t)\left(\eta D_{\eta}^{2}+\gamma D_{\eta}-k^{2}\right), \\
& L_{z}^{(7)}-L_{t}^{(7)}=(z-t)\left(\omega D_{\eta}-1\right) .
\end{aligned}
$$

In both Lemma 2 and Lemma 3 the kernel satisfies an ordinary differential equation of hypergeometric type or simpler type. Now we turn to equations (29) and to the boundary-value problems associated with these self-adjoint forms. In this case, we may seek, instead of an integral relation, an integral equation of Fredholm type

$$
w(z)=\lambda \int_{T_{1}}^{T_{2}} K(z, t) w(t) d t .
$$

It follows from (25), (28), and (34) that

$$
K(z, t)=A(z, t)[G(z)]^{-1}[G(t)]^{-1} .
$$

Suppose that the set of eigenfunctions of (52) is the same as the set of eigenfunctions originated by one of equations (29) on the interval $\left[T_{1}, T_{2}\right]$. Then the following necessary condition, arising from integration by parts in (36),

$$
\left.R(t)\left[w(t) D_{t} K(z, t)-K(z, t) D_{t} w(t)\right]\right|_{T_{1}} ^{T_{2}}=0
$$

should be satisfied. Since equation (54) depends on $z$ as a parameter, the points $T_{1}$ and $T_{2}$ appear to be (if there is no additional symmetry) singularities of the initial equation (29).

\section{Rigorous formulations}

In this section, several theorems are formulated which are by no means exhaustive. The proof of all of them is based on the standard facts about the asymptotic behavior at singularities of solutions of the equations being considered, standard theorems on convergence of integrals depending on a parameter, and properly checking condition (36).

Theorem 1. Suppose that condition (6) for equation (1) is satisfied, and the value of $a$ is negative. Then the boundary conditions

$$
|w(0)|<\infty, \quad|w(1)|<\infty
$$

lead for corresponding equation (29) to a self-adjoint boundary-value problem on the interval $[0,1]$ with the infinite set of eigenfunctions denoted by $\left\{w_{n}(z)\right\}$ and eigenvalues $\left\{q_{n}\right\}$. The set of eigenfunctions serves also as a complete set of eigenfunctions of the integral equation of Fredholm type with continuous symmetrical kernel

$$
w(z)=\lambda \int_{0}^{1}\left[G^{(0)}(z) G^{(0)}(t)\right]^{-1} F\left(\alpha, \beta, \gamma, \frac{z t}{a}\right) w(t) d t
$$

where $F(\alpha, \beta, \gamma, x)$ is the standard hypergeometric function, and the function $G^{(0)}(z)$ is defined by (30). 
This result is a special case of the integral equation obtained in [4].

Integral equations associated with the confluent Heun equation differ first by the kernel and second by the interval of integration.

Theorem 2. Suppose that characteristic exponents $\rho_{2}\left(z_{j}\right)$ and $\beta_{2}(\infty)$ for the equation (10) are chosen as above. Then the boundary conditions

$$
|w(0)|<\infty, \quad|w(1)|<\infty
$$

lead for the corresponding equation (29) to a self-adjoint boundary-value problem, the eigenfunctions $w_{n}(z)$ of which are the same as the eigenfunctions of the two integral equations

$$
w(z)=\lambda \int_{0}^{1}\left[G^{(1)}(z) G^{(1)}(t)\right]^{-1} \Phi(\alpha, \gamma, p z t) w(t) d t
$$

and

$$
w(z)=\mu \int_{0}^{1}\left[G^{(1)}(z) G^{(1)}(t)\right]^{-1} \Phi(\alpha, \gamma+\delta, p(z+t-1)) w(t) d t
$$

where $G^{(1)}(z)$ is defined by (31) and $\Phi$ is the standard confluent hypergeometric function.

Two other integral equations appear when the interval $[0,1]$ is replaced by $[1, \infty]$ and the function $\Phi$ is replaced with the function $\Psi$ - the other standard solution of the confluent hypergeometric equation [3]. Integral equations related to the special case of the confluent Heun equation have been studied in [2].

For the biconfluent Heun equation, we obtain only one integral equation relating to a given interval.

Theorem 3. Suppose that $G^{(2)}(z)$ is the function which transforms equation (14) to the self-adjoint form, and $w_{n}(z)$ satisfies the conditions

$$
|w(o)|<\infty, \quad|w(\infty)|<\infty
$$

Then the eigenfunctions $w_{n}(z)$ are also eigenfunctions of the integral equation

$$
w(z)=\lambda \int_{0}^{\infty}\left[G^{(2)}(z) G^{(2)}(t)\right]^{-1} \Psi\left(\frac{\alpha}{2}, \frac{1}{2} ; \frac{(z+t-\mu)^{2}}{2}\right) w(t) d t
$$

The other integral equation is associated with the boundary-value problem on the interval $[-\infty, 0]$. Instead of examining this case, it is possible to perform the transformation $z \mapsto-z$ in the initial equation. Equation (62) is a simplification of the equation obtained in [8].

The use of Lemma 3 does not lead to an integral equation of Fredholm type in case of arbitrary coefficients in (14) since (54) holds only if the value $\alpha$ is a nonpositive integer. But for this latter special case, we get an integral equation of the form

$$
w(z)=\Lambda \int_{0}^{\infty}\left[G^{2}(z) G^{2}(t)\right]^{-1} \Psi(\alpha, \gamma ;-z t) w(t) d t
$$

where $\Psi$ is the confluent hypergeometric function of the second kind.

In the case of the double-confluent Heun equation, two types of kernels may be used. 
Theorem 4. Suppose that $\nu>0$. The boundary conditions (60) lead to a set of eigenfunctions for the self-adjoint form of the double-confluent Heun equation, which are also eigenfunctions of the integral equations

$$
w(z)=\lambda \int_{0}^{\infty}\left[G^{(3)}(z) G^{(3)}(t)\right]^{-1}(z t+\nu)^{-\alpha} w(t) d t
$$

and

$$
w(z)=\Lambda \int_{0}^{\infty}\left[G^{(3)}(z) G^{(3)}(t)\right]^{-1} \Psi(\alpha, \tau, z+t) w(t) d t .
$$

As far as we know, these equations are new.

In the case of the triconfluent Heun equation, we get no integral equation of Fredholm type related to the corresponding differential boundary problem. The same statement is valid for equation (23).

Theorem 5. Suppose that $\gamma-1>0$. The boundary conditions (60) for the selfadjoint form of equation (21) lead to eigenfunctions $w_{n}(z)$ which satisfy the integral equation

$$
w(z)=\lambda \int_{0}^{\infty}(z t)^{(\gamma-1) / 2} \operatorname{Ai}(z+t-f) w(z) d t
$$

where $\operatorname{Ai}(x)$ is the standard Airy function, namely decreasing at $+\infty$ and solving the equation $y^{\prime \prime}(z)=z y(z)$.

Similar results can be found in [1].

Theorem 6. Suppose that $\omega<0$. Boundary conditions (60) lead for the self-adjoint form of equation (22) to a boundary-value problem on the interval $[0, \infty)$ whose eigenfunctions satisfy the two integral equations

$$
w(z)=\lambda \int_{0}^{\infty}(z t)^{\rho / 2-1} \exp \left(\frac{\omega}{2(1 / z+1 / t)}\right) X(z+t) w(t) d t
$$

and

$$
w(z)=\mu \int_{0}^{\infty}(z t)^{\rho / 2-1} \exp \left(\frac{\omega}{2(1 / z+1 / t)}\right) \exp \left(\frac{z t}{\omega}\right) w(t) d t
$$

where

$$
X(s)=s^{(1-\rho) / 2} K_{1-\rho}(2 \sqrt{s})
$$

and $K_{\mu}(x)$ is the decreasing at $+\infty$ solution of the modified Bessel equation [3].

These equations are new.

Our list of integral equations may be enlarged by several means. Firstly, some more equations can be deduced by our methods if certain parameters of the original differential equation satisfy additional conditions. Secondly, we can test more sophisticated kernels such as $A\left(z^{2}+t^{2}+\kappa z t\right)$.

\section{Proofs}

As written above, the proofs of our theorems can be performed in a unique manner by verifying the convergence of integrals and simple equalities. For this reason, we 
give only one proof for probably the most unusual integral equation (67). By simple substitution, the differential equation for the function $w(z)$ is obtained from (22) as

$$
K w=\frac{d}{d z} z^{2} \frac{d}{d z} w(z)+\left(-\frac{\omega^{2}}{4 z^{2}}-\frac{\omega(1-\rho / 2)}{z}+\frac{\rho(1-\rho / 2)}{2}+q-z\right) w(z)=0 .
$$

The local behavior of the solutions of (69) is

$$
\begin{aligned}
w_{z \rightarrow+0}^{(1)} & =e^{\omega / z} z^{\rho / 2-1}(1+O(z)), \\
w_{z \rightarrow+0}^{(2)} & =e^{-\omega / z} z^{-\rho / 2+1}(1+O(z)), \\
w_{z \rightarrow+\infty}^{(3)} & =e^{-2 \sqrt{z}} z^{-3 / 4}\left(1+O\left(z^{-1}\right)\right), \\
w_{z \rightarrow+\infty}^{(4)} & =e^{2 \sqrt{z}} z^{-3 / 4}\left(1+O\left(z^{-1}\right)\right) .
\end{aligned}
$$

Under our assumption on the parameter $\omega$, only solutions $w^{(1)}, w^{(3)}$ satisfy the boundary conditions (60). By setting Wronskian of these solutions to zero, we get the secular equation for eigenvalues $q_{n}$. Standard theorems on the spectrum of singular differential operators show that there is an infinite set of eigenvalues bounded from below satisfying $q_{n} \rightarrow \infty$ as $n \rightarrow \infty$. The asymptotic estimates (70) for eigenfunctions imply that for any $z \in[0, \infty[$, the integral in the right-hand side of (67) is absolutely and uniformly convergent and equals a function $U(z)$ that is regular on the ray $] 0, \infty[$. Estimates of the integral at small and large $z$ show that this function satisfies the same asymptotic behavior at the end-points as the eigenfunctions of the differential operator functions $w_{n}(z)$.

Now it is only necessary to prove that $U(z)$ satisfies the same differential equation as $w(z)$. It can be easily verified by direct differentiation that $L^{(7)} G=G K$ and $K=K^{*}$ where $G(t)=t^{1-\rho / 2} \exp (-\omega /(2 t))$. The differential operator from (69) acting on the function $U(z)$ on the left-hand side of (67) gives

$$
\begin{aligned}
K_{z} U(z)= & \mu \int_{0}^{\infty}\left[K_{z} G^{-1}(z) \exp \left(\frac{z t}{w}\right)\right] G^{-1}(t) w(t) d t \\
= & \mu \int_{0}^{\infty} G^{-1}(z)\left[L_{z}^{(7)} \exp \left(\frac{z t}{\omega}\right)\right] G^{-1}(t) w(t) d t \\
= & \mu \int_{0}^{\infty} G^{-1}(z)\left[L_{t}^{(7)} \exp \left(\frac{z t}{\omega}\right)\right] G^{-1}(t) w(t) d t \\
= & \mu \int_{0}^{\infty} G^{-1}(z)\left[K_{t} G^{-1}(t) \exp \left(\frac{z t}{\omega}\right)\right] w(t) d t \\
= & \mu G^{-1}(z)\left[t^{2}\left(w(t) \frac{d}{d t} G^{-1}(t) \exp \left(\frac{z t}{\omega}\right)-G^{-1}(t) \exp \left(\frac{z t}{\omega}\right) w^{\prime}(t)\right)\right]_{0}^{\infty} \\
& \quad+\mu \int_{0}^{\infty} G^{-1}(z)\left[G^{-1}(t) \exp \left(\frac{z t}{\omega}\right)\right] K_{t} w(t) d t \\
= & 0
\end{aligned}
$$

All differentiations under the integral sign can be justified, and the non-integral terms vanish on the basis of asymptotic estimates of $w(z)$ at the end-points (70). Since the function $U(x z)$ satisfies the same boundary conditions as $w_{n}(z)$ and the same differential equation, it must be proportional to $w_{n}(z)$. The proportionality factor is, in fact, the eigenvalue $\mu_{n}$. 
The eigenvalue $\mu_{n}$ can be determined by

$$
\mu_{n}=\left[\int_{0}^{\infty} t^{\rho / 2-1} \exp \left(\frac{\omega}{2 t}\right) w_{n}(t) d t\right]^{-1} \lim _{z \rightarrow 0}\left[z^{1-\rho / 2} \exp \left(-\frac{\omega}{2 z}\right) w_{n}(z)\right] .
$$

Acknowledgements. This article has been partly sponsored by the Max-Planck Society. The authors are indebted to Prof. A. Seeger, Prof. D. Schmidt, Dr. W. Lay, and Dr. A. Stahlhofen for helpful discussions. They also thank the referee for a very careful reading of the article which resulted in several improvements.

\section{References}

1. D. I. Abramov, Hydrogen atom in homogeneous electrical field. Integral equations and relations, Sov. J. Vestnik LGU, 1984 (in Russian).

2. D. I. Abramov and I. V. Komarov, Integral equations and relations for Coulomb spheroidal functions, Sov. J. Teoret. i Matem. Fyz. 29 (1976), 235-243.

3. H. Bateman and A. Erdelyi, Higher Transcendental Functions, McGraw Hill, New York, 1953.

4. A. Decarreau, M. C. Dumont-Lepage, P. Maroni, A. Robert, and A. Ronveaux, Formes canoniques des equations confluentes de l'equation de Heun, Ann. Soc. Sc. de Brux. Vol. 92, I-II, pp.53-78, 1978.

5. A. Erdelyi, Integral Equations for Heun Functions, Quart. J. Math. (Oxford) 13 (1942), $107-112$.

6. A. Ya. Kazakov, The Laplace transformation and the differential equations with polynomial coefficients, Sov. J. Differenz. Uravn. 22 (1986), 354-356.

7. C. Markett, Product formulas for eigenfunctions of Sturm-Liouville equations of Heun type, in [2].

8. P. Maroni, Sur quelqes relations integrales entre les solutions de l' equations biconfluente de Heun, Ann. Inst. Henri Poincare 30 (1979), 315-332.

9. D. Schmidt and G. Wolf, $A$ method of generating integral relations by the simultaneous separability of generalized Schrödinger equation, SIAM J. Math. Anal. 10 (1979), 823-838.

10. A. Seeger and W. Lay, Eds., Centennial Workshop on Heun's Equation - Theory and Applications, Max-Planck-Institut für Metallforschung, Stuttgart, 1990.

St. Petersburg State University, Uljanovskaya 1, 198904 St. Petergoff, Russia 